\title{
Separation of Dispersive Coinciding Signals by combining Hard and Soft modeling
}

\author{
Jesper Martinsson, Fredrik Hägglund and Johan E. Carlson \\ EISLAB, Dept. of Computer Science and Electrical Engineering, \\ Luleå University of Technology, SE-971 87 Luleå, Sweden, Jesper.Martinsson@1tu.se
}

\begin{abstract}
In some ultrasonic measurement situations, an adequate signal separation is difficult to achieve. A typical situation is material characterization of thin media, relative to the emitted signal's time support. In this paper a new method is proposed that enables accurate signal separation of measured coinciding signals in the postprocessing stage. The method is based on a combination of hard physical and soft empirical models, which allows for a description of both known and unknown dynamics making the separation possible. The proposed technique is verified using real measurements on thin dispersive samples and validated with residual analysis.
\end{abstract}

Keywords: Signal separation, coinciding signals, overlapping echoes, dispersive media

\section{A. Introduction}

When designing ultrasonic measurement systems based on pulse-echo or through-transmission techniques, adequate signal separation is often a requirement for the analysis step. In flow measurement and material characterization, separable signals are necessary to obtain accurate estimates of transit times and material properties. However, there are a number of factors which can prevent a complete separation. If the propagation distance in the medium is short (due to fixed dimensions or poor signal-to-noise ratios (SNR)), or if the signal's time support is long (due to low-frequency transducers or if high-energy and lowamplitude excitations are required), a complete separation can be difficult to achieve.

For a dispersive media, a separation of coinciding signals can only be obtained if the dynamic properties of the media are known. However, to estimate these properties a complete separation is normally required.

In this paper we use a combination of hard and soft modeling to solve this problem. A hard model structure is applied to describe multiple reflections and overlaps related to the specific measurement setup. A soft model structure is used to capture unknown dispersion and absorption effects from the medium, and to handle unwanted diffraction and misalignment effects from the measurement setup.

The proposed method achieves accurate signal separation in dispersive experimental situations. The proposed technique enables material characterization and flow analysis in otherwise unrealizable situations.

\section{B. Theory}

This section begins with a derivation of the hard model structure for the specific measurement setup shown in Fig. 1. The hard structure describes the ideal case when absorption, dispersion, diffraction, and misalignment effects are neglected and the structure contains only pure delays, reflection and transmission coefficients. This structure describes how the signals overlap and is essential to obtain separation. The hard structure is then expanded with soft structures to cope with a more realistic case when the above effects are included. Finally, details concerning parameterization, parameter estimation, model selection, model validation, and signal separation are given.

\section{B.1. The Hard Model}

Assume that there are no absorption and dispersion effects present, linear acoustic applies, and that the measurement setup is perfectly aligned. Under these assumptions there exist a linear system $\tilde{h}(\omega, \boldsymbol{\theta})$ that describes the relationship between the response $\tilde{y}(\omega)$ and the reference signal $\tilde{u}_{3}(\omega)$ as

$$
\tilde{y}(\omega)=\tilde{h}(\omega, \boldsymbol{\theta}) \tilde{u}_{3}(\omega),
$$

where the response is defined as the summation of all the echoes obtained from the sample space, see Fig. 1,

$$
\tilde{y}(\omega)=\sum_{p=1}^{\infty} \tilde{y}_{p}(\omega),
$$

and the reference signal is defined as the received reflection, $\tilde{u}_{3}(\omega)$, from the water-buffer boundary. Here $\sim$ denotes Fourier transformed quantities and $\omega$ represents the angular frequency. In (1), $\boldsymbol{\theta}$ represents a vector containing the model parameters.

The structure for $\tilde{h}(\omega, \boldsymbol{\theta})$ is found by deriving the expressions for the echoes, $\tilde{y}_{p}(\omega)$, from the sample space using reflection and transmission coefficients related to each layer. The first two contributions,

$$
\begin{aligned}
& \tilde{y}_{1}(\omega)=\frac{R_{12} T_{10} T_{01}}{R_{01}} e^{-j \omega 2 \tau_{1}} \tilde{u}_{3}(\omega) \\
& \tilde{y}_{2}(\omega)=\frac{T_{12} R_{23} T_{21} T_{10} T_{01}}{R_{01}} e^{-j \omega 2\left(\tau_{1}+\tau_{2}\right)} \tilde{u}_{3}(\omega)
\end{aligned}
$$

are unique but the sequential contributions can be expressed in a recursive manner as

$$
\tilde{y}_{p}(\omega)=R_{21} R_{23} e^{-j \omega 2 \tau_{2}} \tilde{y}_{p-1}(\omega), \text { for } p \geq 3 .
$$


Here $R_{l m}$ and $T_{l m}$ denotes the reflection and transmission coefficient respectively, between the $l$ :th and the $m$ :th medium. The phase delay for the wave through the $m$ :th medium is represented by $\tau_{m}$, see Fig. 1 .

The response, $\tilde{y}(\omega)$, in (2) can now be simplified using (5) as

$$
\begin{aligned}
\tilde{y}(\omega) & =\tilde{y}_{1}(\omega)+\sum_{p=2}^{\infty}\left(R_{21} R_{23} e^{-j \omega 2 \tau_{2}}\right)^{p-2} \tilde{y}_{2}(\omega) \\
& =\tilde{y}_{1}(\omega)+\frac{1}{1-R_{21} R_{23} e^{-j \omega 2 \tau_{2}}} \tilde{y}_{2}(\omega)
\end{aligned}
$$

where the summation relationship $\sum_{p=0}^{\infty} x^{p}=1 /(1-x)$, for $|x|<1$, has been used in the last equality.

Combining the expressions in (3), (4) and (6), the hard model structure in (1) can be expressed as

$$
\begin{aligned}
\tilde{h}(\omega, \boldsymbol{\theta})= & \left(R_{12}+\frac{\left(1-R_{12}^{2}\right) R_{23} e^{-j \omega 2 \tau_{2}}}{1+R_{12} R_{23} e^{-j \omega 2 \tau_{2}}}\right) \\
& \times \frac{1-R_{01}^{2}}{R_{01}} e^{-j \omega 2 \tau_{1}}
\end{aligned}
$$

where the relationship, $R_{l m}=-R_{m l}$ and $T_{l m}=1-$ $R_{l m}$, is used and the vector $\boldsymbol{\theta}=\left[R_{01}, R_{12}, R_{23}, \tau_{1}, \tau_{2}\right]^{T}$ represents the unknown model parameters.

\section{B.2. Combining Hard and Soft Modeling}

In cases of diffraction, alignment errors, and frequency dependent absorption and dispersion, the model structure in (7) is inadequate. However, if linear acoustic applies, the above effects can be described using linear systems and (1) is still valid. Assume that a wave that has propagated through the $m$ :th medium is affected by: a system $\tilde{M}_{m}(\omega)$ describing the absorption and dispersion from the medium; and a system $\tilde{D}\left(\omega, \tilde{\xi}_{m}(\omega)\right)$ describing effects that depends on the total number of propagated wave numbers, $\tilde{\xi}_{m}(\omega)=d_{m} \tilde{k}_{m}(\omega) / 2 \pi$, such as diffraction and alignment errors, where $d_{m}$ and $\tilde{k}_{m}(\omega)$ are the thickness and frequency dependent wave number respectively.

Note that in the sequential analysis the $\omega$ dependency

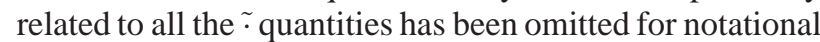
simplicity. Introducing the soft model structures $\tilde{M}_{m}$ and $\tilde{D}(\xi)$, the equations (3), (4) and (5) is expanded to

$$
\begin{aligned}
\tilde{y}_{1}= & \tilde{M}_{1}^{2} \frac{\tilde{D}\left(2 \tilde{\xi}_{1}+\tilde{\xi}_{0}\right) \tilde{D}\left(\tilde{\xi}_{0}\right)}{\tilde{D}\left(2 \tilde{\xi}_{0}\right)} \\
& \times \frac{\tilde{R}_{12} \tilde{T}_{10} \tilde{T}_{01}}{\tilde{R}_{01}} e^{-j \omega 2 \tau_{1}} \tilde{u}_{3}, \\
\tilde{y}_{2}= & \tilde{M}_{1}^{2} \tilde{M}_{2}^{2} \frac{\tilde{D}\left(2 \tilde{\xi}_{1}+2 \tilde{\xi}_{2}+\tilde{\xi}_{0}\right) \tilde{D}\left(\tilde{\xi}_{0}\right)}{\tilde{D}\left(2 \tilde{\xi}_{0}\right)} \\
& \times \frac{\tilde{T}_{12} \tilde{R}_{23} \tilde{T}_{21} \tilde{T}_{10} \tilde{T}_{01}}{\tilde{R}_{01}} e^{-j \omega 2\left(\tau_{1}+\tau_{2}\right)} \tilde{u}_{3}, \\
\tilde{y}_{p}= & \tilde{M}_{2}^{2} \frac{\tilde{D}\left(2 \tilde{\xi}_{1}+(p-1) 2 \tilde{\xi}_{2}+\tilde{\xi}_{0}\right)}{\tilde{D}_{2}\left(2 \tilde{\xi}_{1}+(p-2) 2 \tilde{\xi}_{2}+\tilde{\xi}_{0}\right)} \\
& \times \tilde{R}_{21} \tilde{R}_{23} e^{-j \omega 2 \tau_{2}} \tilde{y}_{p-1}, \text { for } p \geq 3 .
\end{aligned}
$$

In this case the phase velocity and the phase delay are frequency dependent, which means that the reflection and transmission coefficients are frequency dependent. Here $\tau_{m}$ should be considered as the constant phase delay (or dead-time).

Using relatively high frequencies combined with a small sample space ( $\operatorname{small} \widetilde{\xi}_{2}$ ), the diffraction/alignment factor in (10) can be considered as independent of $p$ and approximated as

$$
\frac{\tilde{D}\left(2 \tilde{\xi}_{1}+(p-1) 2 \tilde{\xi}_{2}+\tilde{\xi}_{0}\right)}{\tilde{D}\left(2 \tilde{\xi}_{1}+(p-2) 2 \tilde{\xi}_{2}+\tilde{\xi}_{0}\right)} \approx \tilde{E} .
$$

Using the approximation in (11), the response, $\tilde{y}$, can be expressed analogous to (6) as

$$
\tilde{y}=\tilde{y}_{1}+\frac{1}{1-\tilde{R}_{21} \tilde{R}_{23} \tilde{M}_{2}^{2} \tilde{E} e^{-j \omega 2 \tau_{2}}} \tilde{y}_{2} .
$$

Combining the expressions in (8), (9) and (12), the combined model structure in (1) can be expressed as

$$
\tilde{h}(\boldsymbol{\theta})=\left(\tilde{R}_{12}+\frac{\left(1-\tilde{R}_{12}^{2}\right) \tilde{B} e^{-j \omega 2 \tau_{2}}}{1+\tilde{R}_{12} \tilde{B} e^{-j \omega 2 \tau_{2}}}\right) \tilde{A} e^{-j \omega 2 \tau_{1}},
$$

where

$$
\begin{aligned}
\tilde{A} & =\tilde{M}_{1}^{2} \frac{\tilde{D}\left(2 \tilde{\xi}_{1}+\tilde{\xi}_{0}\right) \tilde{D}\left(\tilde{\xi}_{0}\right)}{\tilde{D}\left(2 \tilde{\xi}_{0}\right)} \frac{\left(1-\tilde{R}_{01}^{2}\right)}{\tilde{R}_{01}} \\
\tilde{B} & =\tilde{M}_{2}^{2} \tilde{E} \tilde{R}_{23} .
\end{aligned}
$$

Normally it is the sample's acoustic properties in $\tilde{M}_{2}$ (and $\tau_{2}$ ) that we wish to estimate. If the sample space is large enough so that a complete separation between each $\tilde{y}_{p}$ is obtained, information of $\tilde{M}_{2}$ can be found using a combination of (8), (9) and (11) as

$$
\tilde{y}_{2}=\frac{\left(1-\tilde{R}_{12}^{2}\right)}{\tilde{R}_{12}} \tilde{B} e^{-j \omega 2 \tau_{2}} \tilde{y}_{1},
$$

and/or (10) and (11) as

$$
\tilde{y}_{p}=-\tilde{R}_{12} \tilde{B} e^{-j \omega 2 \tau_{2}} \tilde{y}_{p-1} .
$$

However, if the sample space is small and a complete separation is difficult to achieved ( $\tilde{y}_{p}$ is unknown), then using (16) or (17) is fruitless. To retrieve information of the sample's acoustic properties the whole model in (13) must be applied, and $\tilde{A}, \tilde{R}_{12}$ and $\tilde{B}$ must be parameterized.

\section{B.3. Parameterization of the Soft Models}

Some of the effects described by the models $\tilde{A}, \tilde{R}_{12}$ and $\tilde{B}$ have unknown physical structures, e.g., diffraction, misalignment, and acoustic properties of the investigated sample. This means that a general structure is needed to parameterize these models. In this paper a phase compensated finite impulse response (FIR) filter is used to parameterize $\tilde{A}, \tilde{R}_{12}$ and $\tilde{B}$ as

$$
\begin{aligned}
\tilde{A}(\mathbf{a}) & =e^{-j \omega T_{s}\left(n_{a}-1\right) / 2} \sum_{p=1}^{n_{a}} a_{p} e^{-j \omega T_{s}(p-1)}, \\
\tilde{R}_{12}(\mathbf{r}) & =e^{-j \omega T_{s}\left(n_{r}-1\right) / 2} \sum_{p=1}^{n_{r}} r_{p} e^{-j \omega T_{s}(p-1)}, \\
\tilde{B}(\mathbf{b}) & =e^{-j \omega T_{s}\left(n_{b}-1\right) / 2} \sum_{p=1}^{n_{b}} b_{p} e^{-j \omega T_{s}(p-1)},
\end{aligned}
$$


where $n_{a}, n_{r}$ and $n_{b}$ are the dimensions of the parameter vectors $\mathbf{a}, \mathbf{r}$ and $\mathbf{b}$ respectively, and $T_{s}$ denotes the sampling period. The term $e^{-j \omega T_{s}(n .-1) / 2}$ represents the phase compensation part and shifts the FIR so that the middle tap corresponds to zero delay (for odd $n$.).

The FIR filter describing the reflection coefficient in (19) is restricted to a type I linear-phase FIR filter [1] with zero phase, i.e., the FIR is symmetric $r_{p}=r_{n_{r}-p+1}$. This means that $\tilde{R}_{12}$ is a real-valued frequency dependent quantity, i.e., no phase shifts other than $\pm \pi$ are associated with reflection and transmission. The linear-phase restriction in (19) can be relaxed, allowing for complex-valued reflection and transmission coefficients, e.g., in case of incomplete or partially contacting interfaces [2], [3].

\section{B.4. Parameter Estimation, Model Selection and Model Validation}

Given data from a pulse-echo experiment, the unknown model parameters are estimated from discrete Fourier transformed data [4], using a nonlinear least-squares (NLS) fit and the Levenberg-Marquardt optimization [5].

To prevent over-parameterization, the minimum description length (MDL) is used [6], [7] to find the appropriate number of FIR parameters. The taps are gradually increased until the MDL is obtained. To reduce the search space, the FIR parameters are not increased independently but simultaneously resulting in a slightly overparameterized model, since the FIR parameters are restricted to equal lengths.

To make sure that the combined model is capable of describing the true system we have to validate its performance. The key concept here is to examine the residuals (the part of the response $y(t)$ that the model could not reproduce) to determine the model's validity [7]. If the residual contain components other than measurement noise, a complete separation can not be justified.

\section{B.5. Signal Separation}

Once the parameter vector $\boldsymbol{\theta}=\left[\mathbf{a}^{T}, \mathbf{r}^{T}, \mathbf{b}^{T}, \tau_{1}, \tau_{2}\right]^{T}$ is estimated and the model is validated, the first signal can be separated using (8) as

$$
\tilde{y}_{1}=\tilde{R}_{12}(\mathbf{r}) \tilde{A}(\mathbf{a}) e^{-j \omega 2 \tau_{1}} \tilde{u}_{3}
$$

and the sequential signals can be found using (21) together with (16) and (17).

\section{Experimental Setup}

The experimental setup consists of a broadband piezoelectric transducer mounted on a custom built measurement cell consisting of a water region and a buffer rod made of $10.030 \pm 0.001 \mathrm{~mm}$ plexiglass. The sample space is located between the buffer rod and a stainless steel reflector, see Fig. 1. A pulser/receiver 5073PR from Panametrics was used to excite the transducer and amplify the received signal. The signal was then digitized using a CompuScope 12400 oscilloscope card, by Gage Applied Technologies Inc., Lachine, QC Canada, at 12-bit resolution and using a sampling rate of $200 \mathrm{MHz}$.

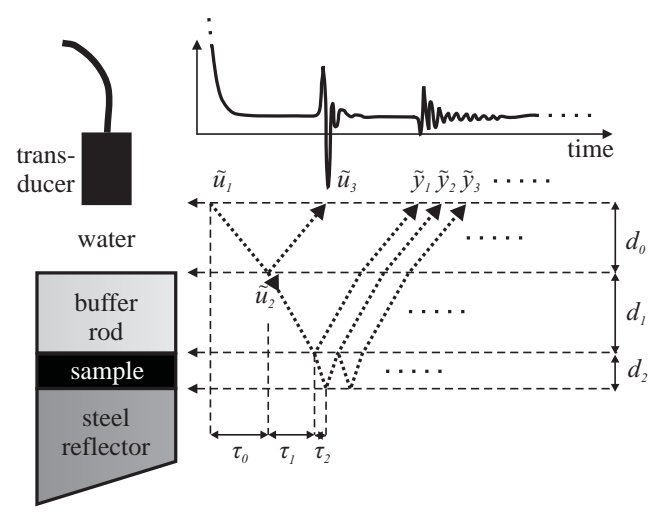

Fig. 1. Schematic figure over the measurement setup and the pulse-echo principle. The transducer emits an unknown sound wave $\tilde{u}_{1}(\omega)$. The reflection from the buffer rod $\tilde{u}_{3}(\omega)$ and the multiple reflections from the sample space $\sum_{p=1}^{\infty} \tilde{y}_{p}(\omega)$ are then recorded. For this particular experiment four different medias has been used: $\tilde{M}_{0}(\omega)$ : water region, $\tilde{M}_{1}(\omega)$ : plexiglas buffer, $\tilde{M}_{2}(\omega)$ : sample, and $\tilde{M}_{3}(\omega)$ : steel reflector.

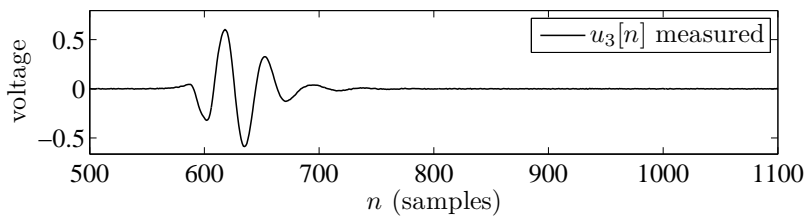

Fig. 2. The measured reference signal $u_{3}[n]$, defined in Section B.1. as the echo from the buffer rod, see Fig. 1.

Three different dispersive test samples were used in this study: petrolatum (vaseline), marine gear oil (SAE90), and glycerine. The pulse-echo tests were performed using four different broadband piezoelectric transducers with center frequencies: $5 \mathrm{MHz}$ (V3456), $10 \mathrm{MHz}$ (V311), $15 \mathrm{MHz}$ (V319), and $20 \mathrm{MHz}$ (V317), manufactured by Panametrics, Waltham, MA, USA. To obtain an adequate SNR (and overlapping echoes), a small sample space was designed using a $0.097 \pm 0.001 \mathrm{~mm}$ washer between the buffer rod and the reflector.

\section{Results}

In this section the estimation, validation and separation results are presented for measurements in petrolatum (most dispersive test sample) with the $5 \mathrm{MHz}$ (V3456) transducer (signal with the longest time support). Similar separation results were obtained for the less dispersive test samples, and with transducers using higher center frequencies.

Fig. 2 shows the measured reference signal using the 5 $\mathrm{MHz}$ transducer. In Fig. 3, estimation results can be seen for two cases. In the first case, represented by the red curve, the hard model structure in (7) is used to estimate the response. In the second case, represented by the blue curve, the combined model structure in (13) is used to estimate the response.

Estimating the response using only the hard model structure leaves a large systematic variation in the residual, Fig. 3 b), indicating presence of un-modeled dynamics originating from diffraction, dispersion, absorption and 


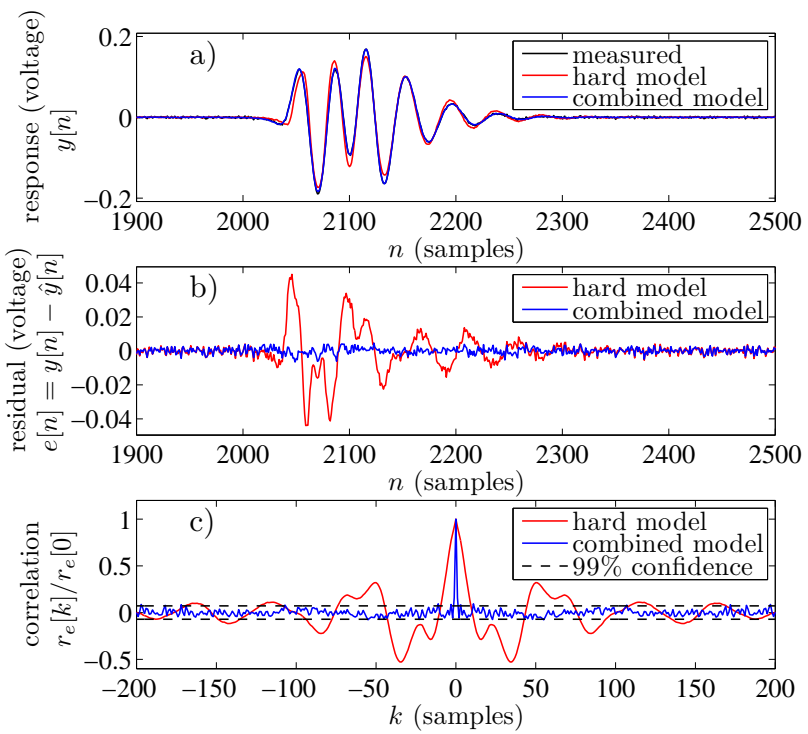

Fig. 3. Estimation results: a) measured response $y[n]$ in black versus the estimated response $\hat{y}[n]$ using (7) in red and using (13) in blue; b) the residuals; $c$ ) whiteness test of the residuals, the dashed lines mark the $99 \%$ confidence region for $k \neq 0$.

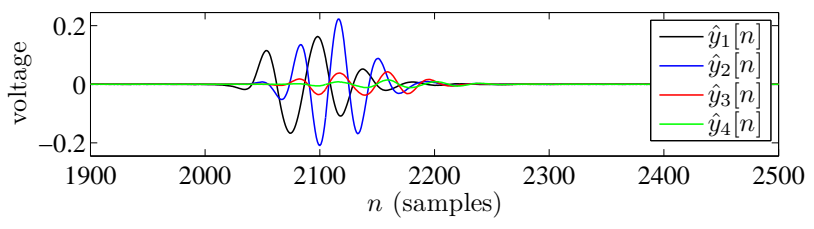

Fig. 4. Separation of the first four coinciding signals using the estimated parameters from the combined model structure, see Section B.5..

alignment errors neglected by the model, discussed in Section B.1.. This is also indicated by a highly autocorrelated residual in Fig. 3 c), where the tails $(k \neq 0)$ clearly violates the confidence region [7]. Since the hard model structure can not describe the true system, an adequate separation using this structure can not be achieved. However, using the combined model structure (13) a good fit can be seen in Fig. 3 a) and only a slight systematic variation is visible in the residual in Fig. 3 b). The results are presented for the case when $n_{a}=n_{r}=n_{b}=19$ given by the MDL criteria, Section B.4.. The visible variation can be reduced further if the model order is allowed to increase, however, the MDL criteria and the residual analysis presented in Fig. 3 c) indicates that the appropriate model order is found and the residual correlation falls within the confidence region. Once the parameter vector is estimated and the model is validated, the coinciding signals can be separated, see Fig. 4, using the relationships derived in Section B.5..

\section{E. Discussion}

The assumption that the residual is uncorrelated (white) is only valid for situations where the measurement noise on reference signal is negligible. In presence of considerable reference noise, a natural correlation appears even though a good model is used. However, if it is possible to con- duct repeated experiments with the same excitation signal, the covariance matrix can be estimated from the repeated experiments and an errors-in-variables (EV) approach can be applied [4]. The EV approach offers also an extended residual analysis that includes noisy reference signal situations.

The theory presented in this paper is derived for the specific measurement setup shown in Fig. 1, but the ideas of combining hard and soft modeling can naturally be rewritten for other measurement setups or extended to apply for multi-layered material/media. The parameterization of the soft models can be changed to a more physical structure based on the physical properties of the media, if the diffraction/alignment factor can be neglected. If a more restrictive structure is used it is important to remember the model validation step to justify a complete separation. Another alternative is to first use the more general FIR representation to obtain a complete separation, and then fit a physical structure to the frequency response of the FIR filters.

\section{F. Conclusions}

In this paper we use a combination of hard and soft modeling to separate coinciding dispersive ultrasonic signals. A hard model structure is derived from the physical properties of the measurement setup, and contains the basic structure which enables separation. The hard structure is expanded with soft models, in the form of FIR filters, offering a general structure to cope with dispersion, absorption, diffraction and alignment errors from the measurement setup. The proposed model's ability to separate coinciding signals is demonstrated for measurements on thin dispersive samples and is validated using residual analysis. The experimental results show that accurate separation is achieved with uncorrelated residuals.

\section{G. Literature}

[1] B. Porat, A Course In Digital Signal Processing. John Wiley and Sons, 1997.

[2] K. Kendall and D. Tabor, "An ultrasonic study of the area of contact between stationary and sliding surfaces," in Proceedings of the Royal Sociey, Series A, pp. 321-340, 1971.

[3] B. W. Drinkwater, R. S. Dwyer-Joyce, and P. Cawley, "A study of the interaction between ultrasound and a partially contacting solid-solid interface," in Proceedings of the Royal Sociey, Series A, pp. 2613-2628, 1996.

[4] R. Pintelton and J. Schoukens, System Identification: A Frequency Domain Approach. IEEE Press, 2001.

[5] R. Fletcher, Practical Methods of Optimization. New York: Wiley, 2nd ed., 1991.

[6] J. Rissanen, "Modelling by the shortest data description," Automatica, vol. 14, pp. 465-471, 1978.

[7] L. Ljung, System Identification: Theory for the User. Englewood Cliffs: Prentice-Hall, 2nd ed., 1987. 